\title{
Rhodnius prolixus and $R$. robustus (Hemiptera: Reduviidae) nymphs show different locomotor patterns on an automated recording system
}

\author{
Márcio G. Pavan ${ }^{1,2^{*}}$, Jessica Corrêa-Antônio ${ }^{1}$, Alexandre A. Peixoto ${ }^{2,3^{\wedge}}$, Fernando A. Monteiro ${ }^{1,3 \dagger}$
} and Gustavo B. S. Rivas $2,4^{*}+$

\begin{abstract}
Background: Circadian rhythms of triatomines, vectors of the etiological agent Trypanosoma cruzi responsible for Chagas disease, have been extensively studied in adults of the two most epidemiologically relevant vector species, Rhodnius prolixus and Triatoma infestans. However, little attention has been dedicated to the activity patterns in earlier developmental stages, even though triatomine nymphs are equally capable of transmitting T. cruzi to humans. Because circadian rhythms may differ even between closely related species, studies that focus on this behavioral trait can also be used to shed light on the taxonomy of controversial taxa, which becomes especially relevant regarding vector species.

Methods: We compared the daily locomotor activity patterns of second- and third-instar nymphs of Rhodnius prolixus and Rhodnius robustus in order to unveil possible behavioral differences between these cryptic species. Mitochondrial and nuclear markers were sequenced to confirm species identification.

Results: Nymphs of both species had a bimodal pattern of locomotion and similar daily activity patterns, but $R$. prolixus is more active under light/dark cycles and depicts a more pronounced activity rhythm under constant darkness conditions.

Conclusions: We describe the implementation of an often-used automated method for the recording of individual locomotor activity to differentiate sibling species of Rhodnius with distinct epidemiological relevance. The higher levels of activity observed in the nymphs of $R$. prolixus could potentially contribute to increased vector capacity.
\end{abstract}

Keywords: Rhodnius prolixus, Rhodnius robustus, Triatominae, Rhodnius, Locomotor activity, Chronobiology, Behaviour, Cryptic species

\section{Background}

American trypanosomiasis (Chagas disease) is a neglected vector-borne disease caused by Trypanosoma cruzi (Kinetoplastida: Trypanosomatidae) and transmitted to humans mainly via infected feces of triatomine bugs (Hemiptera: Reduviidae). It is believed that 6-7 million people are estimated to be infected, mostly in Latin

\footnotetext{
*Correspondence: marciopavan@gmail.com; gbsrivas@gmail.com

${ }^{\dagger}$ Equal contributors

Deceased

'Laboratório de Epidemiologia e Sistemática Molecular, Instituto Oswaldo Cruz, FIOCRUZ, Rio de Janeiro, Brazil

2Laboratório de Biologia Molecular de Insetos, Instituto Oswaldo Cruz,

FIOCRUZ, Rio de Janeiro, Brazil

Full list of author information is available at the end of the article
}

America and the Caribbean [1]. Disease prevention principally depends upon eliminating household-infesting triatomines since there is no vaccine or practical treatment on a large scale [2].

Rhodnius prolixus and Rhodnius robustus (sensu lato) (s.l.) are members of a cryptic species complex that includes at least six different lineages [3]. While $R$. prolixus is the main domestic Chagas disease vector in Colombia, Venezuela and certain areas of Central America $[4,5], R$. robustus lineages are exclusively sylvatic and distributed throughout the Amazon region [3]. Although it is relatively well established that $R$. prolixus and $R$. robustus (s.l.) are bona fide species based on genetic markers [3, 6-8], 
no behavioral or morphological evidence has ever been produced to back up the molecular evidence.

The circadian control of insect activity rhythms $[9,10]$ is an area of behavioral science particularly relevant for the understanding of epidemiologically-related questions as it affects the time and the degree of contact between either the vector and the host or the vector and insecticidesprayed surfaces $[11,12]$. Moreover, behavioral studies conducted on sibling species might help identify evolutionary processes responsible for the formation of natural reproductive isolation barriers for gene exchange [13, 14].

Circadian rhythms of triatomines have been extensively studied in adults of the two most epidemiological relevant vector species, $R$. prolixus and Triatoma infestans. In both species these rhythms seem to control basic biological processes such as reproduction, foraging, breeding, oviposition [15, 16], dispersion [17] and host-seeking [18, 19]. Besides the light-dark and temperature cycles [20], the presence of a host seems to be an important zeitgeber [21]. As a foraging strategy, $R$. prolixus and T. infestans spend the day in refuges that are out of predator reach (in vertebrate nests and burrows in sylvatic habitats, or cracks and crevices when inside human dwellings) and become active at night when they leave to blood feed [22]. It is also known that $R$. prolixus males will also readily leave their shelters induced by the presence of females [23]. The nocturnal activity patterns displayed by adult triatomine bugs are generally bimodal. The first peak occurs just after dusk and corresponds to host-seeking activities, whereas the second is at dawn, interpreted as an effort by the insect to search for an appropriate daytime shelter [20, 22, 24].

Despite extensive studies on the locomotor activity patterns of adult $R$. prolixus and T. infestans, little attention has been dedicated to the activity patterns in earlier developmental stages even though triatomine nymphs are equally capable of transmitting $T$. cruzi to humans.

Locomotor activity rhythm is governed by molecular mechanisms, which in turn are regulated by a number of clock genes $[25,26]$. Thus, interspecific differences in activity patterns should indicate evolutionary divergence [13]. Because circadian rhythms differ even between closely related species [13, 27], studying these rhythms may help to resolve controversial taxonomic issues. Therefore, the aim of the current work is to compare the daily locomotor activity patterns of second- and third-instar nymphs of $R$. prolixus and $R$. robustus in order to unveil possible behavioral differences between these cryptic species.

\section{Methods}

\section{Species identification}

We used in all assays laboratory-reared $R$. prolixus and $R$. robustus (s.l.) collected in Guatemala (Apr/2004) and Peru (June/1994), respectively. Since these are cryptic species, the identity and purity of colonies were confirmed through DNA sequence analyses and phylogenetic reconstructions. Twenty specimens of each colony were sequenced for two molecular markers: (i) a 264-bp fragment of the fourth intron of the single-copy nuclear Transmembrane protein 165 gene (TPS165), referred to as AmpG, that contains a SNP that differentiates $R$. prolixus from $R$. robustus (s.l.) [8] and (ii) a 682-bp fragment of the mitochondrial cytochrome $b$ gene $(c y t b)$, which differentiates not only $R$. prolixus from $R$. robustus (s.l.), but also discriminates all four cryptic species of $R$. robustus complex ( $R$. robustus I-IV) [3, 6].

DNA samples were extracted from 1 to 2 legs of each specimen using the Promega kit, according to the manufacturer's recommendations. cytb and AmpG fragments were amplified as previously described $[6,8]$. Purification of PCR products was carried out with the Hi Yield ${ }^{\mathrm{Tm}} \mathrm{Gel} /$ PCR DNA Extraction Kit (Real Genomics ${ }^{\mathrm{Tm}}$ ). Forward and reverse strands were subjected to fluorescent dyeterminator cycle sequencing reactions (ABI Prism ${ }^{\circledR}$ BigDye $^{\circ}$ Terminator v3.1 Cycle Sequencing Kit, Applied Biosystems) and run on an ABI 3730 automated sequencer.

Bayesian phylogenetic trees were reconstructed according to Abad-Franch et al. [3] and Pavan et al. [8] (see Additional file 1 for detailed specifications).

\section{Locomotor activity recording}

Second- and third-instar nymphs of $R$. prolixus and $R$. robustus (s.l.) fed on chicken blood ten days prior to experimentation. Only nymphs not fed to repletion were selected, and specimens that moulted until the date of the experiment were discarded. Insects were individually transferred to $1 \times 7 \mathrm{~cm}$ glass tubes with both ends properly sealed with Parafilm ${ }^{\circ}$ M (Sigma-Aldrich). Locomotor activity analyses were performed with a larger version of the Drosophila DAM5 Activity Monitoring System (Trikinetics Inc., Waltham, MA, USA), as previously described [13, 28]. This system incorporates an infrared interruption method that detects movement every time the insect crosses the beam. Each movement detected by the monitor was recorded with computer software (DAMSystem3 Software).

All monitors were placed in a Precision Scientific Incubator (Model 818), under a relative humidity of $75 \%$ at constant temperature of $25{ }^{\circ} \mathrm{C}$. Activities were recorded under an artificial photoperiodic regime of 12:12 LD (cycles of 12-h of light and 12-h of darkness) for five days, followed by 23 days of 24-h DD (constant darkness) and five days of 12:12 LD. The experimental procedure was performed in triplicate. These data were pooled as a preliminary statistical analysis and showed no significant differences between the repetitions.

Graph comparison of daily activity patterns between $R$. prolixus and $R$. robustus in 12:12 LD was performed in Excel $^{\mathrm{TM}}\left(\right.$ Microsoft $\left.{ }^{\odot}\right)$, depicting the average activity of five 
days under this regime. Weighted moving averages of data were used to "smooth" data variation. Only insects with noticeable activity lasting for at least six consecutive days were analyzed. For these triatomines, the total activity under 12:12 LD was estimated by summing the locomotion recorded during five consecutive days. In parallel, we also calculated the proportion of nocturnal activity, dividing the sum of activity recorded during the dark phase by the total amount of daily activity, as previously described [13]. Proportions were normalized by the arcsine square root method in order to be further analyzed with parametric statistics. Comparisons were conducted based on the Analysis of variance (ANOVA).

After five days under 12:12 LD, all nymphs were submitted to 23 days of constant dark conditions (DD). Under this regime, triatomines normally depict a free-running pattern of locomotor activity which represents an apparent persistence of the endogenous rhythm and may differ from the $24-\mathrm{h}$ period. To determine this pattern, all individual actograms were smoothed (smoothing Gaussian standard deviation tool in software ActogramJ) to reduce the noise observed in the periodograms [29]. Then, the freerunning period length $(\tau)$ of each insect was calculated with the $\chi^{2}$ periodogram algorithm [30] in ActogramJ [29]. The free-running period length was calculated in three different time windows: (i) considering the first ten days $\left(\tau_{1-10}\right)$ which was pointed out by Refinetti [31] as being optimal; (ii) the last thirteen days $\left(\tau_{11-23}\right)$ which depicts any uncoupling effect of oscillators due to long starvation period; and (iii) all 23 days under DD $\left(\tau_{23}\right)$, aiming to define if longer days in DD could increase the resolution of the analysis.

We estimated the strength (power) of the rhythm which is defined as the amplitude from the top of the peak to the confidence level in the $\chi^{2}$ periodogram [32]. Liu et al. [32] previously showed that some per mutants of Drosophila are arrhythmic, and therefore, their "power" is zero. Also, other minor impairments on per expression into the brain of Drosophila specimens could affect the strength of rhythm, which could be visualized by lower power levels compared to wild-type flies [32]. More recently, this method has been adopted to evaluate the effect that the knockdown of clock genes has on the rhythm of non-Drosophila species [33-35]. Therefore, estimates of power levels are effective for evaluating the rhythmicity of such organisms and can be useful to depict possible differences among Rhodnius species under laboratory conditions.

\section{Actogram plotting and acrophase calculation}

Only insects that demonstrated continuous activity throughout all the experiments and a $\chi^{2}$ periodogram with a peak above the confidence level were selected to represent average actograms of $R$. robustus(s.l.) and $R$. prolixus. From the average actogram, we calculated the acrophase (with the acrophase tool in ActogramJ), a parameter that indicates the central phase of the activity cycle which generally coincides with the main peak of activity [36].

\section{Statistical analysis}

Statistical analysis and graphs were accomplished with the GraphPad Prism 5 package (Prism, La Jolla, CA), as previously described [34, 37]. We checked if all parameters analyzed followed a Gaussian distribution with the Shapiro-Wilk normality test $(P<0.05)$. Only data from the 23-day free-running treatment under DD $\left(\tau_{23}\right)$ passed this test and thus, were submitted to the parametric unpaired Student's $t$ test (with $5 \%$ significance level). The other parameters $\left(\tau_{1-10}, \tau_{11-23}\right.$ and "power" level) were analyzed with the Mann-Whitney test $(P<0.05)$. The proportion of nocturnal activity and the total daily activity were statistically compared between the two species. Oneway ANOVA was performed for this comparison in the $\mathrm{R}$ environment [38].

\section{Results \\ Species identification}

Phylogenetic analyses confirmed the identification of the specimens used as $R$. prolixus and $R$. robustus II (sensu Pavan et al. [8]). The $20 R$. prolixus specimens had the same mitochondrial cytb haplotype and nuclear AmpG genotype as EF011726 and JQ432888, specimens collected in Venezuela and Guatemala, respectively [7, 8]. The other 20 specimens analyzed had the same haplotype and genotype of $R$. robustus II from Napo, Ecuador (AF421341 and JQ432896, respectively) [7, 8] (Fig. 1).

\section{Locomotor activity pattern}

When submitted to light/dark cycles (12:12 LD, 1000 lux), nymphs of $R$. robustus II and $R$. prolixus showed similar activity patterns throughout the day and shared the same circadian pattern (Fig. 2). At dusk, individuals of both species increased their activity one hour before lights on/off transition, leading to a peak at early night. Then, locomotion starts to decrease until basal levels throughout the night. The highest levels of activity were recorded during this interval (ZT11-ZT14) and, together with night activity, represent the major part of daily activity in both species (58.2\% $R$. robustus and $53.9 \%$ for $R$. prolixus). Oneway ANOVA comparing the proportion of activity during scotophase revealed that there was no difference between species $\left(F_{(1,135)}=0.38 ; P=0.538\right)$. Also, 

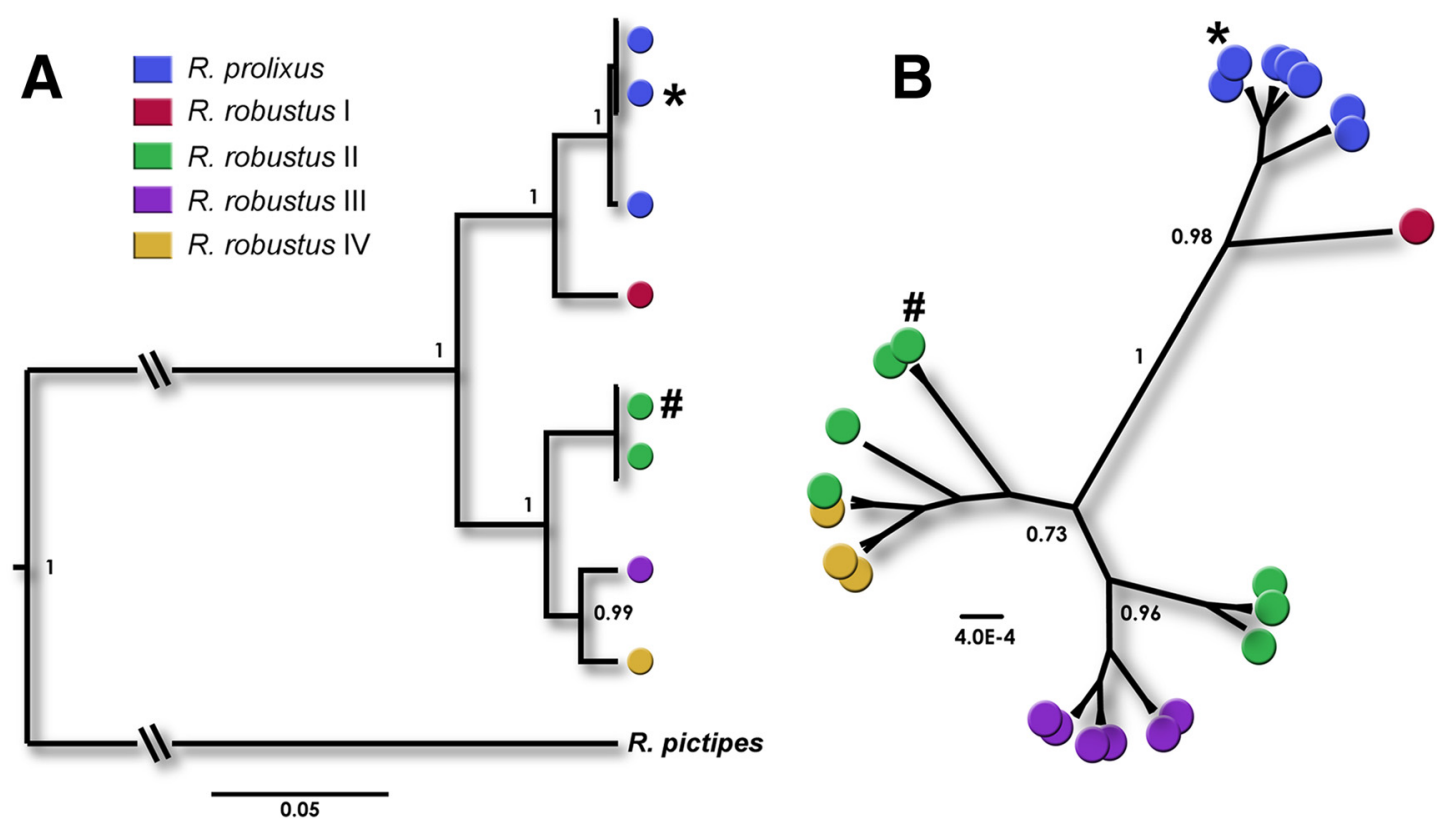

Fig. 1 Phylogenetic analyses of R. prolixus and R. robustus (s.l.) Bayesian consensus trees of 9 cytb (a) and 24 AmpG sequences (b). Branches that connect $R$. prolixus $+R$. robustus and $R$. pictipes were reduced for a better visualization. Posterior probabilities $>0.7$ are shown for key nodes. Node circles represent sequences of $R$. prolixus and R. robustus (s.l.) and are color-coded (see color key). The position of $R$. prolixus and $R$. robustus $I$ sequences were highlighted with symbols "**" and "\#", respectively

we observed a minor peak at morning followed by a discrete activity during daytime.

The main difference between species in the 12:12 LD condition concerned the total amount of activity, with $R$. prolixus being noticeably more active than $R$. robustus II (one-way ANOVA $F(1,135)=19.90 ; P<$ 0.001; Fig. 3).

\section{Locomotor activity under constant dark cycles}

$R$. prolixus and $R$. robustus II specimens were also submitted to a regime of complete darkness (DD) for 23 days followed by an additional five days of 12:12 LD at 1000 lux. Figures $4 \mathrm{a}$ and b reveal that the overall activity of both species in darkness is much lower than the previous activity in 12:12 LD, being barely

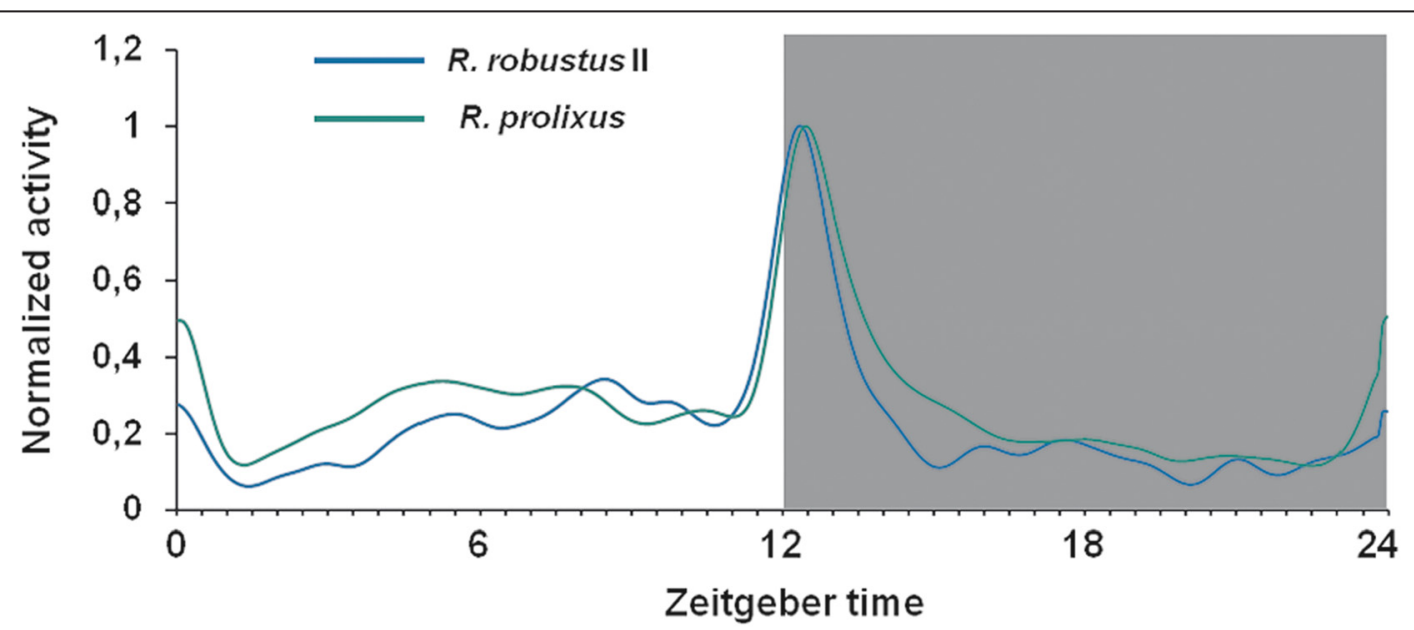

Fig. 2 Daily activity profile of $R$. robustus $\|$ and $R$. prolixus under 12:12 LD. Average locomotor activity of $R$. robustus $\|$ (blue lines, $N=30$ ) and $R$. prolixus (green lines, $N=65$ ), depicted by average values of five days of recording. The horizontal white bar represents the light phase and the black bar indicates the dark phase. ZT = Zeitgeber Time within a light/dark cycle experiment. ZT 0 corresponds to the lights-on event and ZT 12 to the lights-off event 


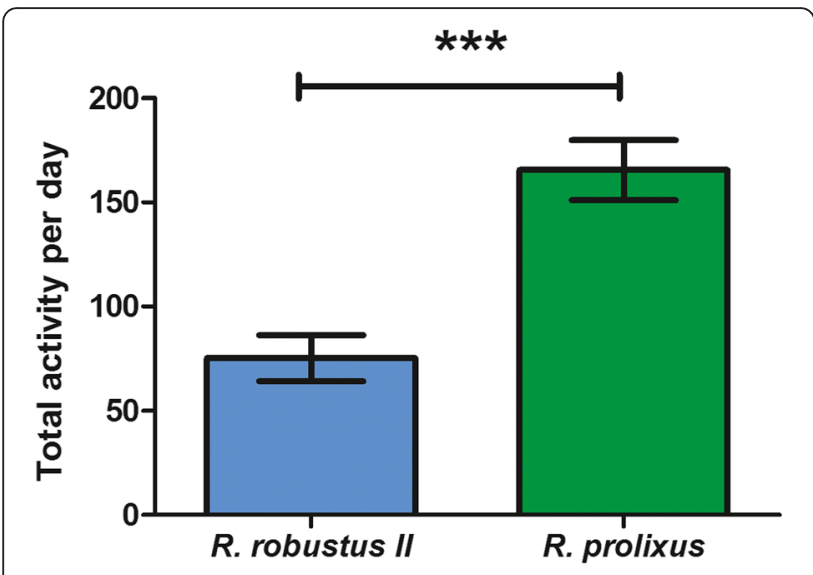

Fig. 3 Locomotor activity levels of R. robustus II and R. prolixus under 12:12 LD. Total locomotor activity per day of $R$. robustus II (blue bar, $N=30$ ) and $R$. prolixus (green bar, $N=65$ ), calculated with five days of recording. Mann Whitney $t$ test $\left({ }^{* *}\right) P<0.001$

visible in actograms. Although reduced, the locomotor activity under DD is rhythmic in both species and presents a circadian period shorter than $24 \mathrm{~h}$ (Fig. 4c and Table 1).

Figure 4d displays a pattern of phase advance during the first 10 days. In the following days, the acrophase of both species started to be inconsistent, exhibiting phase shifts. These inconsistencies seem to appear earlier in $R$. prolixus than in $R$. robustus. This pattern of free running (especially into the last days in DD) strongly suggests that oscillators (i.e. endogenous clocks) have become decoupled in both species. Therefore, we calculated the free-running period based on three time-frames: the whole 23 day period under DD (Fig. 4e), the first 10 days (Fig. 4f) and also the last 13 days (Fig. 4g). Only the calculations of the last 13 days resulted in significant differences in the free-running period between species (Fig. 4f and Table 1). Although this observation could be interpreted as a further evidence of interspecific difference, we feel that it could also have resulted from the decoupling oscillators.

Although free-running periods were similar in both species, more $R$. prolixus specimens were rhythmic and presented higher power levels than $R$. robustus II (Table 1). We observed, by visual inspection of individual periodograms, that some insects depicted a clear free-running pattern under constant darkness. In these cases, the power levels were always above 500 (see Additional file 2). On the other hand, other rhythmic triatomines presented a more complex free-running pattern and power levels below 500 (Additional file 2). Therefore, we decided to classify them into two groups defined as "strongly rhythmic" and "weakly rhythmic". The power levels in Table 1 clearly display a larger number of strongly rhythmic $R$. prolixus specimens. This demonstrates that $R$. prolixus has a more evident behavioral rhythm than $R$. robustus II under constant conditions.

\section{Discussion}

This paper compares the daily activity rhythms between nymphs of the sibling Chagas disease vector species $R$. prolixus and $R$. robustus II, under laboratory conditions. The most salient features observed were the higher activity levels and more pronounced rhythmicity of $R$. prolixus. Although earlier results based on mitochondrial and nuclear markers strongly indicate that $R$. prolixus and $R$. robustus II are valid species [3, 6-8], here we present the first behavioral evidence in support for their separation based upon molecularly certified insects.

Previous behavioral analyses in triatomines have successfully used ingenious systems to record locomotor activity (e.g. [20, 39]). Herein, we evaluated the individual circadian patterns of two Rhodnius species with a commercial automated recording system of locomotor activity widely used in insect chronobiology studies [13, 27, 40-42]. Automated systems are better suited for the collection and compilation of behavioral data since activity is not recorded by visual inspection, therefore potentially minimizing human error.

Behavioral analyses under artificial conditions are more suitable to unveil species-specific patterns that are driven by the endogenous clock [13] since insect activity under natural conditions is influenced by multiple factors, such as social and host cues as well as environmental oscillations. Our results depicted the patterns of daily activity expected for both Rhodnius spp., with two major peaks of activity: one at dawn, most likely associated with the search for refuge, and the other at dusk, related to hostseeking behavior [20, 22]. Besides main peaks, we also observed in both species a basal activity, irrespective of light presence. Although fifth-instar nymphs and adult triatomines seem to have a negative phototactic response [22], Rhodnius second- and third-instar nymphs did not show reduced activity during the light phase.

The daytime activity recorded here varies among bugs, with some presenting small and sparse bouts of activity and others with continuous and high mobility levels. This activity is clearly clock-controlled, because it persisted visibly in constant darkness for more than one week and free-ran with a period of less than $24 \mathrm{~h}$ (Fig. 4c). In any case, it seems that the daytime inactivity in Rhodnius nymphs is fragmented and discontinued. Other insects such as Apis mellifera and Drosophila also alternate states of deep sleep and wakefulness during the day $[43,44]$. In the case of the former, sleep behavior has an ontogenetic dependence. Foragers (old bees) exhibit a nighttime consolidated sleep $[45,46]$, while 


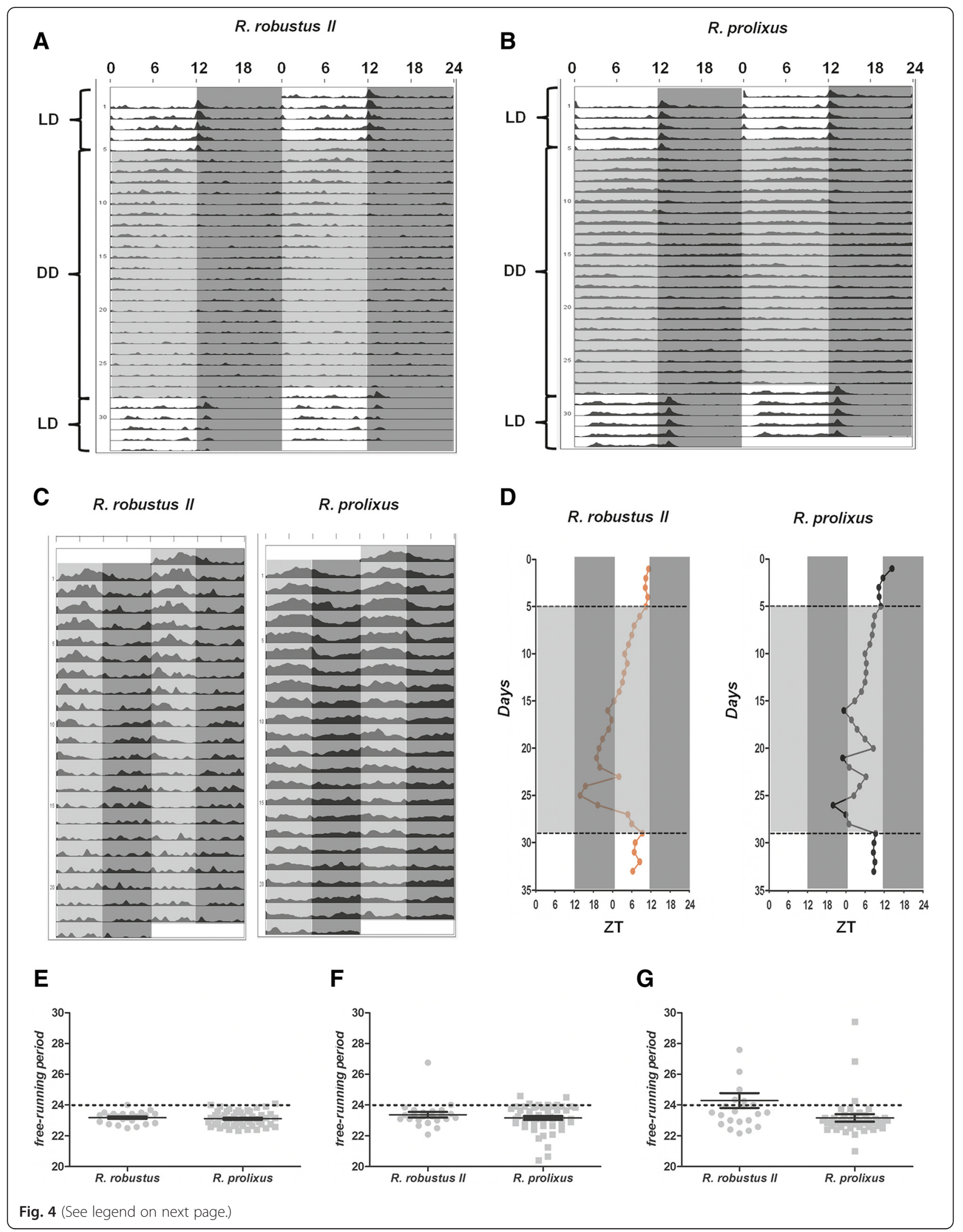


(See figure on previous page.)

Fig. 4 Locomotor activity/rest behavior of R. robustus II and R. prolixus under 12:12 LD followed by constant darkness (DD). Double-plotted actograms for $R$. robustus $I I(\mathbf{a} ; N=23)$ and $R$. prolixus $(\mathbf{b} ; N=50)$ depicting five consecutive days under 12:12 LD followed by 23 days under constant darkness and reverting to another five days under 12:12 LD. c Smoothed double-plotted actograms of both species under constant darkness. The shaded dark grey areas indicate dark phase and light grey areas indicate the subjective days in constant darkness. $\mathbf{d}$ Acrophase of $R$. robustus II (red dotted lines) and $R$. prolixus (black dotted lines) during the 33 days of experiment. Dashed lines represent the transition from LD to DD (top) and from DD to LD (bottom). e-g Free running period length of R. robustus II (circles) and R. prolixus (squares), based on the whole 23-day period under DD (e), the first 10 days (f) and the last 13 days (g). Solid lines represent the average \pm SEM. Dotted lines mark the 24-h period length

young bees distribute their sleep throughout the day $[47,48]$. We cannot discard the hypothesis that light avoidance in nymphs of Rhodnius is influenced by an ontogenetic effect, with younger nymphs being less sensitive to light than older nymphs and adults. Future experiments should help clarify this particular issue.

The results of power levels of activity in constant darkness clearly showed that $R$. prolixus has a circadian locomotor pattern more pronounced than $R$. robustus II. Moreover, nymphs of $R$. prolixus were more rhythmic than nymphs of $R$. robustus II, being able to sustain similar period lengths along the 23-day complete dark condition (Table 1). We suggest that these species have circadian pacemaker differences (i.e. different regulation of gene circadian expression and/or protein abundance). It is well known that circadian clock and metabolism are interconnected in many insect species [49]. Recent RNA interference work based on the knockdown of the clock gene timeless in Aedes aegypti and Gryllus bimaculatus showed that the reduction of tim expression impairs the circadian activity. This impairment weakens the behavioral response of the endogenous clock, which is marked by lower power levels in specimens injected with dstim RNA $[34,50]$. In addition, blood-feeding reduces circadian expression of period, timeless and Clock, which is followed by lower levels of locomotor activity in Aedes aegypti, Aedes albopictus, and Lutzomyia longipalpis [28, 51]. Therefore, we suggest that the higher activity under LD and strong rhythmicity of $R$. prolixus might be a result of the circadian clock and metabolism acting together. In this perspective, we can hypothesize that $R$. prolixus digests blood faster than $R$. robustus II, as previously shown for morphologically identified $R$. prolixus and $R$. robustus (s.l.) from Venezuela [52, 53]. Hence, $R$. prolixus would search for blood meals more frequently, increasing the risk of transmission of T. cruzi.

This is the first study to show behavioral differences between genetically certified $R$. prolixus and $R$. robustus II, two sibling triatomine species of clearly distinct epidemiological relevance. In this respect, we speculate if behavioral differences between these two species could have an impact on their vectorial capacities. Recent

Table 1 Locomotor activity rhythms of R. robustus II and R. prolixus in constant darkness

\begin{tabular}{|c|c|c|c|c|c|}
\hline & & \multicolumn{2}{|l|}{ Species } & \multicolumn{2}{|c|}{ Mann-Whitney test } \\
\hline & & R. robustus II & R. prolixus & $\bar{U}$ & $P$ \\
\hline$\overline{\mathrm{N}}$ & & 33 & 64 & - & - \\
\hline \multirow[t]{3}{*}{ Proportion of rhythmic insects (\%) } & Total & 69.70 & 78.13 & - & - \\
\hline & $S R^{a}$ & 21.21 & 46.88 & - & - \\
\hline & $W R^{\mathrm{a}}$ & 48.48 & 31.25 & - & - \\
\hline \multirow[t]{3}{*}{ Free-running period length $(\text { Mean } \pm S D)^{b}$} & $\tau_{23}$ & $23.17 \pm 0.07$ & $23.10 \pm 0.06$ & NS & NS \\
\hline & $T_{1-10}$ & $23.37 \pm 0.17$ & $23.15 \pm 0.12$ & NS & NS \\
\hline & $\tau_{11-23}$ & $24.29 \pm 0.48$ & $23.16 \pm 0.24$ & 347.5 & 0.0069 \\
\hline \multirow[t]{3}{*}{ Power levels (Mean $\pm S D)^{c}$} & Power 23 & $606.4 \pm 71.96$ & $799.0 \pm 59.53$ & 381.5 & 0.0219 \\
\hline & Power ${ }_{1-10}$ & $329.5 \pm 37.19$ & $472.3 \pm 41.27$ & 379.5 & 0.0206 \\
\hline & Power 11-23 & $330.7 \pm 34.97$ & $398.7 \pm 29.04$ & NS & NS \\
\hline
\end{tabular}

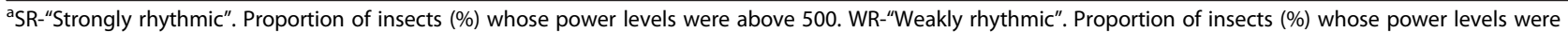
below 500. See results for details

${ }^{\mathrm{b}}$ Free-running period length (in hours \pm SEM) was calculated in three different time windows: (i) considering all 23 days under DD ( $\left.\tau_{23}\right)$; (ii) the first ten days ( $\tau_{1-}$ $\left.{ }_{10}\right)$; and (iii) the last thirteen days $\left(\mathrm{T}_{11-23}\right)$. See Methods for details

${ }^{c}$ The power of the rhythm was defined as the amplitude from the peak to the cutoff line $(a=0.05)$ in the $x^{2}$ periodogram (Liu et al. [32]). See methods for details. The power level was calculated in three different time windows. Power ${ }_{23}$ : considering all 23 days under DD. Power ${ }_{1-10}$ : the first ten days. Power ${ }_{11-23}$ : the last thirteen days

NS,- non-significant 
work on mosquitoes might contribute for a better understanding of this idea. For instance, it was observed that two cryptic species of Anopheles gambiae exhibit similar patterns of flight activity, but one form is more active than the other [27]. The authors suggest that the different levels of activity between these species could affect host-seeking dispersal. Also, a previous work incorporating mathematical modeling indicated a positive correlation between locomotor activity and biting rate in Aedes aegypti [54]. Altogether, we believe that higher levels of activity in $R$. prolixus could contribute to an increase in its dispersal capabilities and blood feeding efficiency thus rendering it a more effective vector.

\section{Conclusions}

We describe the implementation of an often-used automated method for the recording of individual locomotor activity to differentiate sibling Rhodnius spp. with distinct epidemiological relevance. The higher levels of activity and rhythmicity observed in $R$. prolixus nymphs could potentially contribute to increased vector capacity.

\section{Ethical approval}

The animals used to maintain the insects at FIOCRUZ were treated according to the Ethical Principles in Animal Experimentation approved by the Ethics Committee in Animal Experimentation (CEUA/FIOCRUZ) approved under the protocol numbers P-54/10-4/LW12/11 and L0061/08. Both protocols are from CONCEA/MCT (http:// www.cobea.org.br), which is associated with the American Association for Animal Science (AAAS), Federation of European Laboratory Animal Science Associations (FELASA), International Council for Animal Science (ICLAS) and Association for Assessment and Accreditation of Laboratory Animal Care International (AAALAC).

\section{Additional files}

Additional file 1: Protocol S1. Detailed methods used in phylogenetic reconstructions of Rhodnius prolixus and R. robustus. (DOCX $29 \mathrm{~kb}$ )

Additional file 2: Individual actograms with different free-running patterns under constant darkness. A) Double-plotted actogram of a single nymph with a free-running pattern less than $24 \mathrm{~h}$ (left), which is easily observed by visual inspection and confirmed by $x^{2}$ periodogram (right). The periodogram has a peak at $1405 \mathrm{~min}(\sim 23 \mathrm{~h})$ and the $P$ value level for the actogram is displayed as a red line. The "power" value is defined as the amplitude of the peak, and is measured from the confidence level to the top. B) Double-plotted actogram of a single nymph, displaying a more difficult pattern to track by visual inspection (left). The $x^{2}$ periodogram (right) has a peak of 1420 min (approximately 23 h). However, the graph is quite inconsistent and power level is considerably lower than depicted in A. Light and dark grey areas indicate the subjective day and night, respectively, in constant darkness. (TIF 733 kb)

\section{Competing interests}

The authors declare that they have no competing interests.

\section{Authors' contributions}

Conceived and designed the study: MGP, AAP, FAM, GBSR. Performed and analyzed behavioral data: MGP, AAP, GBSR. Performed and analyzed molecular data: MGP, JCA. Drafted the manuscript: MGP, FAM, GBSR. All authors but AAP approved the final version of the manuscript.

\section{Acknowledgements}

We thank Robson Costa da Silva for expert technical assistance and Luciana Araripe for critical revision of the manuscript. We also thank the PDTISFIOCRUZ DNA sequencing core for running sequencing reactions. MGP, JCA, and GBSR received scholarships from the Brazilian funding agencies CAPES, FAPERJ, and CNPq, respectively. Funders had no role in study design, data collection and analysis, decision to publish, or preparation of the manuscript. We dedicate this paper to the memory of Dr. Alexandre Afranio Peixoto, a great mentor and friend.

\section{Author details}

${ }^{1}$ Laboratório de Epidemiologia e Sistemática Molecular, Instituto Oswaldo Cruz, FIOCRUZ, Rio de Janeiro, Brazil. 'Laboratório de Biologia Molecular de Insetos, Instituto Oswaldo Cruz, FIOCRUZ, Rio de Janeiro, Brazil. ${ }^{3}$ Instituto Nacional de Ciência e Tecnologia em Entomologia Molecular (INCT-EM)/ CNPq, Rio de Janeiro, Brazil. ${ }^{4}$ Present address: Laboratório de Bioquímica e Fisiologia de Insetos, Instituto Oswaldo Cruz, FIOCRUZ, Rio de Janeiro, Brazil.

Received: 22 January 2016 Accepted: 30 March 2016

Published online: 27 April 2016

\section{References}

1. WHO: Chagas disease (American trypanosomiasis). Fact sheet 340.2015. http://www.who.int/mediacentre/factsheets/fs340/en/.

2. Abad-Franch F, Santos WS, Schofield CJ. Research needs for Chagas disease prevention. Acta Trop. 2010;115(1-2):44-54.

3. Abad-Franch F, Pavan MG, Jaramillo-O N, Palomeque FS, Dale C, Chaverra D, Monteiro FA. Rhodnius barretti, a new species of Triatominae (Hemiptera: Reduviidae) from western Amazonia. Mem Inst Oswaldo Cruz. 2013;108 Suppl 1:92-9.

4. Fitzpatrick S, Feliciangeli MD, Sanchez-Martin MJ, Monteiro FA, Miles MA. Molecular genetics reveal that silvatic Rhodnius prolixus do colonise rural houses. PLoS Negl Trop Dis. 2008;2(4):e210.

5. Guhl F, Pinto N, Aguilera G. Sylvatic triatominae: a new challenge in vector control transmission. Mem Inst Oswaldo Cruz. 2009;104 Suppl 1:71-5.

6. Monteiro FA, Barrett TV, Fitzpatrick S, Cordon-Rosales C, Feliciangeli D, Beard CB. Molecular phylogeography of the Amazonian Chagas disease vectors Rhodnius prolixus and R. robustus. Mol Ecol. 2003;12(4):997-1006.

7. Pavan MG, Monteiro FA. A multiplex PCR assay that separates Rhodnius prolixus from members of the Rhodnius robustus cryptic species complex (Hemiptera: Reduviidae). Trop Med Int Health. 2007;12(6):751-8.

8. Pavan MG, Mesquita RD, Lawrence GG, Lazoski C, Dotson EM, Abubucker S, Mitreva M, Randall-Maher J, Monteiro FA. A nuclear single-nucleotide polymorphism (SNP) potentially useful for the separation of Rhodnius prolixus from members of the Rhodnius robustus cryptic species complex (Hemiptera: Reduviidae). Infect Genet Evol. 2013;14:426-33.

9. Clements AN. The Biology of Mosquitoes. Wallingford: CABl; 1999.

10. Saunders DS. Insect Clocks. 3rd ed. Amsterdam: Elsevier; 2002.

11. Lazzari CR, Lorenzo MG. Exploiting triatomine behaviour: alternative perspectives for their control. Mem Inst Oswaldo Cruz. 2009;104 Suppl 1:65-70.

12. Marques MD. Biological rhythms and vector insects. Mem Inst Oswaldo Cruz. 2013:108 Suppl 1:59-62.

13. Rivas GB, Souza NA, Peixoto AA. Analysis of the activity patterns of two sympatric sandfly siblings of the Lutzomyia longipalpis species complex from Brazil. Med Vet Entomol. 2008;22(3):288-90.

14. Tauber E, Roe H, Costa R, Hennessy JM, Kyriacou CP. Temporal mating isolation driven by a behavioral gene in Drosophila. Curr Biol. 2003;13(2):140-5.

15. Constantinou C. Circadian Rhythms in Insects. London: University of London; 1979.

16. Lazzari CR. Circadian rhythm of egg hatching in Triatoma infestans (Hemiptera: Reduviidae). J Med Entomol. 1991;28(5):740-1.

17. McEwen PK, Lehane MJ. Factors influencing flight initiation in the triatomine bug Triatoma infestans (Hemiptera: Reduviidae). Insect Sci Applications. 1993;14:461-4. 
18. Barrozo RB, Minoli SA, Lazzari CR. Circadian rhythm of behavioural responsiveness to carbon dioxide in the blood-sucking bug Triatoma infestans (Heteroptera: Reduviidae). J Insect Physiol. 2004;50(2-3):249-54.

19. Bodin A, Barrozo RB, Couton L, Lazzari CR. Temporal modulation and adaptive control of the behavioural response to odours in Rhodnius prolixus. J Insect Physiol. 2008;54(9):1343-8.

20. Lazzari C. Circadian organization of locomotion activity in the haematophagous bug Triatoma infestans. J Insect Physiol. 1992;38:895-903.

21. Valentinuzzi VS, Amelotti I, Gorla DE, Catalá SS, Ralph MR. Circadian entrainment by light and host in the Chagas disease vector, Triatoma infestans. Chronobiol Int. 2014;31(2):265-75.

22. Lorenzo MG, Lazzari CR. Activity pattern in relation to refuge exploitation and feeding in Triatoma infestans (Hemiptera: Reduviidae). Acta Trop. 1998; 70(2):163-70.

23. Pontes G, Zacharias CA, Manrique G, Lorenzo MG. Female odours promote the activation of sheltered kissing bug Rhodnius prolixus males and modulate their orientation. Med Vet Entomol. 2014;28(3):257-63.

24. Settembrini BP. Circadian rhythms of locomotor activity in Triatoma infestans (Hemiptera: Reduviidae). J Med Entomol. 1984;21(2):204-12.

25. Allada R, Chung BY. Circadian organization of behavior and physiology in Drosophila. Annu Rev Physiol. 2010;72:605-24.

26. Hardin PE. Molecular genetic analysis of circadian timekeeping in Drosophila. Adv Genet. 2011;74:141-73.

27. Rund SS, Lee SJ, Bush BR, Duffield GE. Strain- and sex-specific differences in daily flight activity and the circadian clock of Anopheles gambiae mosquitoes. J Insect Physiol. 2012;58(12):1609-19.

28. Meireles-Filho AC, Meireles-Filho AC, da S Rivas GB, Gesto JS, Machado RC, Britto C, e Souza NA, Peixoto AA. The biological clock of an hematophagous insect: locomotor activity rhythms, circadian expression and downregulation after a blood meal. FEBS Lett. 2006;580(1):2-8.

29. Schmid B, Helfrich-Förster C, Yoshii T. A new ImageJ plug-in "ActogramJ" for chronobiological analyses. J Biol Rhythms. 2011;26(5):464-7.

30. Sokolove PG, Bushell WN. The chi square periodogram: its utility for analysis of circadian rhythms. J Theor Biol. 1978;72(1):131-60.

31. Refinetti R, Lissen GC, Halberg F. Procedures for numerical analysis of circadian rhythms. Biol Rhythm Res. 2007:38(4):275-325.

32. Liu X, Yu QA, Huang ZS, Zwiebel LJ, Hall JC, Rosbash M. The strength and periodicity of $D$. melanogaster circadian rhythms are differentially affected by alterations in period gene expression. Neuron. 1991;6(5):753-66.

33. Moriyama Y, Kamae Y, Uryu O, Tomioka K. gb'clock is expressed in the optic lobe and is required for the circadian clock in the cricket Gryllus bimaculatus. J Biol Rhythms. 2012;27(6):467-77.

34. Gentile C, Sehadova H, Simoni A, Chen C, Stanewsky R. Cryptochrome antagonizes synchronization of Drosophila's circadian clock to temperature cycles. Curr Biol. 2013;23(3):185-95.

35. Uryu O, Karpova SG, Tomioka K. The clock gene cycle plays an important role in the circadian clock of the cricket Gryllus bimaculatus. J Insect Physiol. 2013;59(7):697-704.

36. Díez-Noguera A. Methods for serial analysis of long time series in the study of biological rhythms. J Circadian Rhythms. 2013;11(1):7.

37. Rivas GB, de Souza NA, Peixoto AA, Bruno RV. Effects of temperature and photoperiod on daily activity rhythms of Lutzomyia longipalpis (Diptera: Psychodidae). Parasit Vectors. 2014;7:278.

38. Team RDC. R: A Language and Environment for Statistical Computing. Vienna: R Foundation for Statistical Computing; 2010

39. Marliére NP, Latorre-Estivalis JM, Lorenzo MG, Carrasco D, Alves-Silva J, Rodrigues JO, Ferreira LL, Lara LM, Lowenberger C, Guarneri AA. Trypanosomes modify the behavior of their insect hosts: effects on locomotion and on the expression of a related Gene. PLoS Negl Trop Dis. 2015;9(8):e0003973.

40. Rosato E, Kyriacou CP. Analysis of locomotor activity rhythms in Drosophila. Nat Protoc. 2006:1(2):559-68.

41. Gentile C, Rivas GB, Meireles-Filho AC, Lima JB, Peixoto AA. Circadian expression of clock genes in two mosquito disease vectors: cry2 is different. J Biol Rhythms. 2009;24(6):444-51.

42. Giannoni-Guzmán MA, Avalos A, Marrero Perez J, Otero Loperena EJ, Kayım M, Medina JA, Massey SE, Kence M, Kence A, Giray T, et al. Measuring individual locomotor rhythms in honey bees, paper wasps and other similar-sized insects. J Exp Biol. 2014:217(Pt 8):1307-15.

43. Hendricks JC, Finn SM, Panckeri KA, Chavkin J, Williams JA, Sehgal A, Pack Al. Rest in Drosophila is a sleep-like state. Neuron. 2000;25(1):129-38.
44. Kaiser W, Steiner-Kaiser J. Neuronal correlates of sleep, wakefulness and arousal in a diurnal insect. Nature. 1983:301(5902):707-9.

45. Kaiser WJ. Busy bees need rest, too. J Comp Physiol A. 1988;163:565-84.

46. Sauer S, Kinkelin M, Herrmann E, Kaiser W. The dynamics of sleep-like behaviour in honey bees. J Comp Physiol A Neuroethol Sens Neural Behav Physiol. 2003;189(8):599-607.

47. Eban-Rothschild AD, Bloch G. Differences in the sleep architecture of forager and young honeybees (Apis mellifera). J Exp Biol. 2008;211(Pt 15): 2408-16.

48. Klein BA, Olzsowy KM, Klein A, Saunders KM, Seeley TD. Caste-dependent sleep of worker honey bees. J Exp Biol. 2008:211(Pt 18):3028-40.

49. Saunders DS, Hong S. Effects of temperature and temperature-steps on circadian locomotor rhythmicity in the blow fly Calliphora vicina. J Insect Physiol. 2000;46(3):289-95.

50. Danbara Y, Sakamoto T. Uryu O, Tomioka K. RNA interference of timeless gene does not disrupt circadian locomotor rhythms in the cricket Gryllus bimaculatus. J Insect Physiol. 2010;56(12):1738-45.

51. Lima-Camara TN, Lima JB, Bruno RV, Peixoto AA. Effects of insemination and blood-feeding on locomotor activity of Aedes albopictus and Aedes aegypti (Diptera: Culicidae) females under laboratory conditions. Parasit Vectors. 2014;7:304.

52. Sant'Anna MRV, Diotaiuti L, Gontijo AF, Gontijo NF, Pereira MH. Feeding behaviour of morphologically similar Rhodnius species: influence of mechanismal characteristics and salivary function. J Insect Physiol. 2001;47: 1459-65.

53. Aldana E, Lizano $\mathrm{E}$, Rodriguez $\mathrm{M}$, Valderrama A. Alimentación y defecación en triatominos del género Rhodnius (Hemiptera, Reduviidae) alimentados com sangre humana. Rev Biol Trop. 2001:49:693-6.

54. Luz PM, Lima-Camara TN, Bruno RV, Castro MG, Sorgine MH, Lourenço-deOliveira R, Peixoto AA. Potential impact of a presumed increase in the biting activity of dengue-virus-infected Aedes aegypti (Diptera: Culicidae) females on virus transmission dynamics. Mem Inst Oswaldo Cruz. 2011;106(6):755-8.

\section{Submit your next manuscript to BioMed Central and we will help you at every step:}

- We accept pre-submission inquiries

- Our selector tool helps you to find the most relevant journal

- We provide round the clock customer support

- Convenient online submission

- Thorough peer review

- Inclusion in PubMed and all major indexing services

- Maximum visibility for your research

Submit your manuscript at www.biomedcentral.com/submit 